\title{
Optic nerve compression in allergic fungal sinusitis
}

\author{
A THAKAR, P LAL, M DHIWAKAR, S BAHADUR \\ Department of Otolaryngology and Head-Neck Surgery, All India Institute of Medical Sciences, Ansari Nagar, \\ New Delhi, India
}

\begin{abstract}
Objective: To describe the syndrome of optic nerve involvement in cases of allergic fungal sinusitis. Setting: Academic tertiary care centre.

Methods: Analysis of prospectively accrued data for 10 consecutive cases (three bilateral, giving 13 eyes) with visual loss secondary to optic nerve compression, from a total of 70 cases of allergic fungal sinusitis undergoing surgical treatment between June 1997 and May 2007.

Results: The mean duration of rhinological symptoms prior to the onset of visual loss was 22 months. At presentation, visual loss ranged from a visual acuity of 6/12 to complete loss of light perception. Bilateral involvement was noted in three of the 10 cases. Urgent surgical decompression with removal of all fungal debris and decompression of the optic nerve resulted in visual recovery in seven of the 13 eyes. On univariate analysis, recovery was less likely in cases with long standing visual loss, and in cases with complete visual loss.

Conclusion: Long standing allergic fungal sinusitis may be complicated by visual loss due to compression of the optic nerve. Urgent surgery to clear the sinuses and decompress the optic nerve is successful in reversing visual loss in cases with partial visual loss.
\end{abstract}

Key words: Visual Acuity; Fungi; Sinusitis; Hypersensitivity; Surgery

\section{Introduction}

Allergic fungal sinusitis was first described in the late 1970 s and early 1980 s by Safirstein ${ }^{1}$ and Millar et $a l .{ }^{2}$ and was subsequently characterised by Bent and Kuhn. ${ }^{3}$ It is being increasingly diagnosed, and is estimated to account for approximately 7 per cent of all chronic sinusitis cases requiring surgery. ${ }^{4}$ Orbital involvement is infrequent (18 per cent) in reports from the western hemisphere, ${ }^{5}$ but is reported much more frequently in South-East Asian series, with a prevalence as high as 57 per cent. $^{6}$

Proptosis is the commonest orbital manifestation of allergic fungal sinusitis. ${ }^{7}$ Other reported orbital manifestations include diplopia, ophthalmoplegia and visual loss. Visual loss secondary to allergic fungal sinusitis is usually secondary to involvement of the optic nerve, consequent to bony erosion of the lateral sphenoid wall. The condition can be challenging to treat but is fortunately rare, with only a few published reports. $^{5-13}$

We encountered optic nerve involvement leading to visual loss in 10 cases of allergic fungal sinusitis treated over a 10-year period from June 1997 to May 2007. Our experience with these cases is reported.

\section{Materials and methods}

Over a 10-year period from June 1997 to May 2007, 70 cases of allergic fungal sinusitis fulfilling the Bent and Kuhn criteria ${ }^{3}$ were surgically treated by the authors at a tertiary care university referral hospital. Ten of these 70 cases (14 per cent) presented with visual loss consequent to involvement of the optic nerve.

All pre- and post-treatment data pertaining to these patients were prospectively collected on a specifically designed pro forma.

All subjects underwent detailed otolaryngological and ophthalmological evaluation. Ophthalmic examination included visual acuity testing, visual field examination and fundus evaluation. Visual fields were checked by formal perimetry in eight eyes. No fieldcharting could be undertaken in five eyes because of inadequate vision (i.e. visual acuity less than 1/60). All other causes of visual loss were excluded.

Non-contrast computed tomography scanning of the paranasal sinuses, orbit and adjacent skull base delineated the extent of disease.

All patients underwent urgent functional endoscopic sinus surgery or external sphenoethmoidectomy, under general anaesthesia. Polyps and characteristic greenish 


\begin{tabular}{lc}
\multicolumn{2}{c}{ TABLE I } \\
\multicolumn{2}{c}{ CONVERSION FROM VISUAL ACUITY TO LOG MAR } \\
SCALE \\
\hline Visual acuity* ${ }^{*}$ Log MAR \\
\hline Pl -ve & -4.70 \\
Pl +ve & -3.70 \\
Hand movements & -2.70 \\
$1 / 60$ & -1.70 \\
$2 / 60$ & -1.40 \\
$3 / 60$ & -1.30 \\
$6 / 60$ & -1.00 \\
$6 / 30$ & -0.70 \\
$6 / 24$ & -0.60 \\
$6 / 18$ & -0.48 \\
$6 / 12$ & -0.30 \\
$6 / 9$ & -0.18 \\
$6 / 6$ & -0.00 \\
$6 / 5$ & 0.12 \\
$6 / 4$ & 0.18
\end{tabular}

Percentage improvement $=(($ post-operative $\log$ MAR - preoperative log MAR) / (premorbid log MAR - pre-operative log MAR) $\times 100 \%$. * Measured by Snellen's chart. LogMAR $=\log$ of the minimum angle of resolution; PL $-\mathrm{ve}=$ (perception of light)-negative; $\mathrm{PL}+\mathrm{ve}=($ perception of light)-positive

brown debris were removed from the involved nasal cavities and sinuses, and optic nerve decompression was carried out. The removed material was examined histopathologically and showed abundant mucin with eosinophils and Charcot-Layden crystals and also multiple hyphal forms of fungi, confirming the diagnosis of allergic fungal sinusitis.

Post-operatively, patients were placed on a tapering dose of oral prednisolone for one month (beginning with $40 \mathrm{mg} /$ day), and also received steroid nasal drops and nasal irrigation. Regular follow up was scheduled at one, three and six months and one year post-operatively. Every follow-up visit included nasal endoscopic evaluation and visual acuity testing.

To enable quantitative assessment of the degree of visual improvement following surgery, patients' visual acuity results were converted to the $\log$ MAR scale (i.e. $\log$ of the minimum angle of resolution), as per the conversion chart shown in Table I, and the percentage improvement determined. The percentage improvement was calculated as the quantum of improvement following surgery as a proportion of the pre-operative visual deficit (i.e. post-operative vision - pre-operative vision / pre-injury vision - pre-operative vision $\times 100$ per cent). ${ }^{14}$ It was presumed that the premorbid visual acuity was $6 / 6$ (with correction). The visual acuity recorded at the three month postoperative visit was taken to represent the final, maximal improvement.

\section{Results}

The study group of 10 cases comprised six males and four females, aged from 17 to 50 years (mean, 25.3 years). All were immunocompetent.

All patients reported sino-nasal symptoms, such as nasal obstruction, rhinorrhoea and headaches, for a

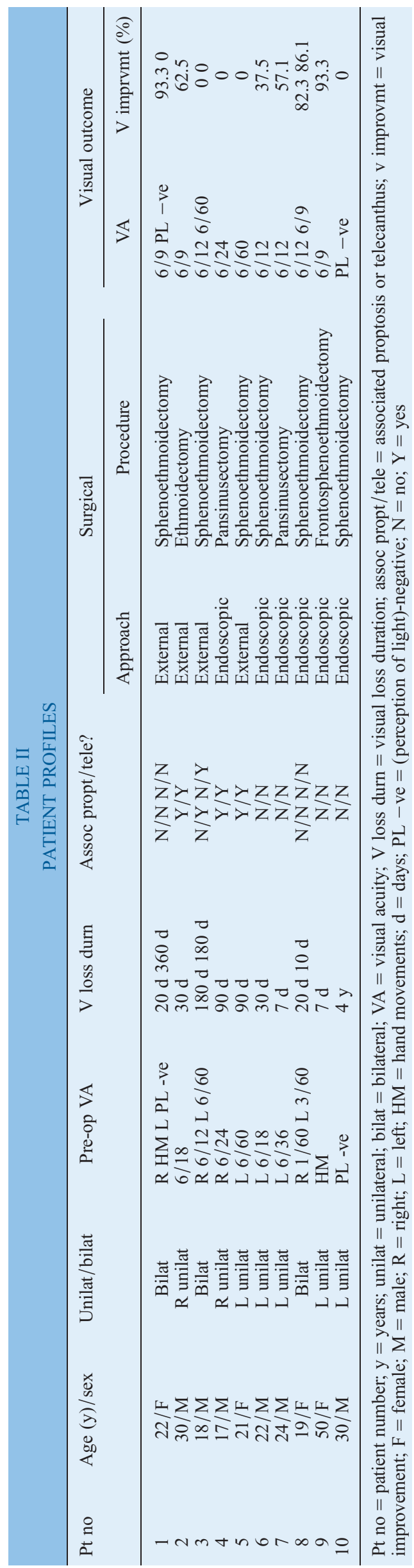


period ranging from three months to five years (mean symptom duration, 22 months). Nasal polyps were present in all cases.

Visual loss was bilateral in three cases and unilateral in seven cases. The duration of visual loss ranged from four days to four years (median duration, 30 days). Visual acuity at presentation ranged from $6 / 12$ to (perception of light)-negative (Table II). Fundus evaluation indicated optic atrophy in four cases. Three out of 13 eyes showed proptosis, and five cases had telecanthus.

Radiology demonstrated the characteristic hyperattenuating foci seen in allergic fungal sinusitis, along with expansion and destruction of the sinus walls. Erosion of the lateral wall of the sphenoid and the optic canal was noted in all patients. Lateral and superior expansion of the sphenoid sinus and spread of the disease beyond its boundaries so as to extend to the middle cranial fossa were noted in five cases (Figures 1 and 2). Associated extension to the anterior cranial fossa was noted in one case.

Four of the 10 cases underwent surgery via the external approach, while the remaining underwent endoscopic surgery. Surgery involved complete clearance of the sinus debris and further decompression of the optic nerve. Disease extension to the anterior and middle cranial fossa (always extradural) was completely cleared. In all instances, the medial wall of the optic canal had been partially or completely eroded by the disease so as to expose the nerve on the lateral wall of the sinus. Surgical removal of allergic and fungal debris in the sphenoid sinus itself relieved pressure on the nerve. The nerve was also surgically decompressed along the entire length of the optic canal by removing the intervening bone between the nerve and the sphenoid sinus lumen. The nerve was exposed from the optic ring anteriorly to its medial bend posteriorly. The medial bend of the nerve is taken to signify the transition of the nerve from the canal-encased canalicular segment to the free-lying intracranial segment, and is thus taken as the posterior

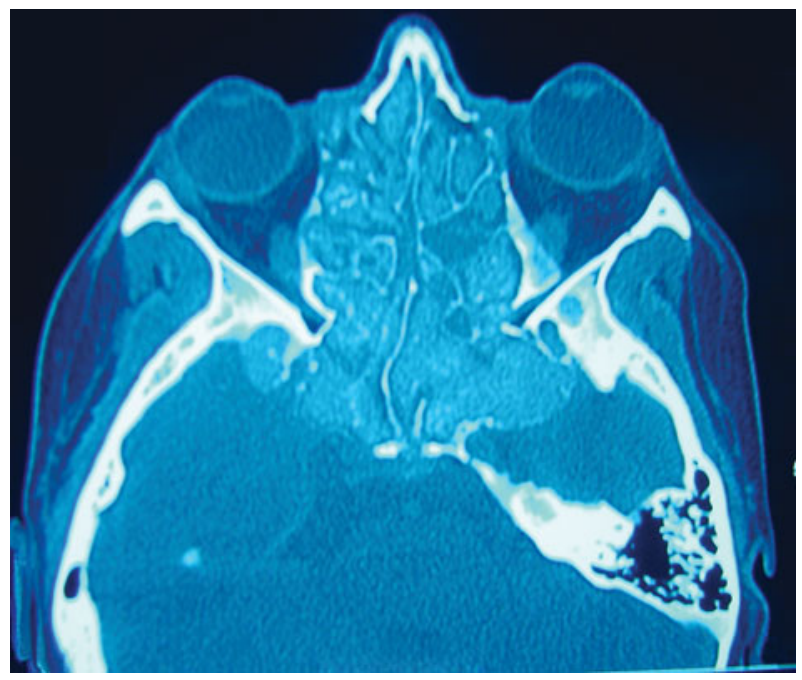

FIG. 1

Axial computed tomography scan showing characteristic hyperattenuating foci, indicative of allergic fungal debris, in the ethmoid and sphenoid sinuses, with expansion of the sinuses and compression and splaying of both optic nerves, and also extension into the middle cranial fossa.

limit of decompression. ${ }^{14}$ The nerve sheath was not incised.

Table II shows our patients' post-operative visual acuity and the degree of recovery (percentage improvement) at three months. Visual recovery was noted in seven of the 13 eyes (54 per cent). Initial improvement was noted within a few days of decompression, with continuing improvement occurring until approximately six weeks post-operatively. All seven eyes with recent onset visual loss (i.e. $<30$ days), even if severe (i.e. hand movements, $1 / 60$ or $3 / 60$ vision), demonstrated visual improvement following surgical decompression. However, none of the six eyes with visual loss of greater duration demonstrated any improvement. In the cases that improved, the quantum of visual improvement ranged from 37.5 to 93.3 per cent, with the post-operative visual acuity in all being $6 / 12$ or (a)

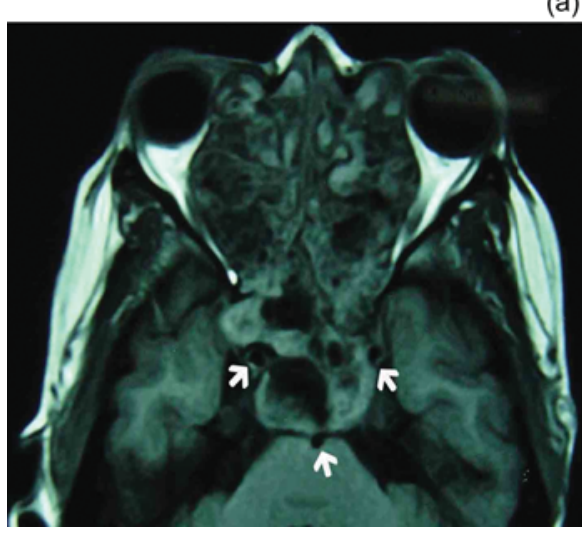

(b)

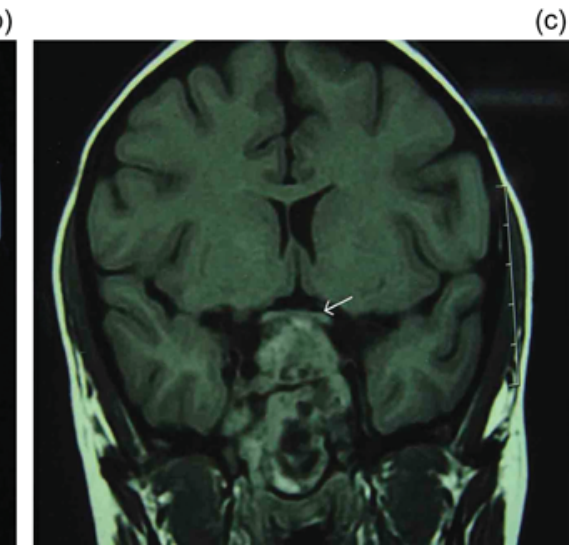

FIG. 2

Axial (a) and coronal (b \& c) T1-weighted magnetic resonance imaging scans, demonstrating ethmoidal expansion with compression of the orbit and intraorbital optic nerve (a), and sphenoidal expansion with the sinus contents abutting the optic nerve and chiasma (thin arrows) and also the internal carotid and basilar arteries (thick arrows). 
better; however, no case returned to $6 / 6$ vision. Two cases had complete visual loss (i.e. (perception of light)-negative) dating from one year and four years, variously; neither of these cases showed any post-operative visual recovery.

\section{Discussion}

Visual loss secondary to optic nerve involvement has been infrequently noted in allergic fungal sinusitis cases, with only a few reports, as shown in Table III. The current paper reports 10 such cases (representing 13 eyes, as three cases had bilateral involvement), and notes a good potential for recovery with surgical decompression provided it is undertaken soon after the onset of visual loss. However, cases with a delay of more than 30 days after the onset of visual loss, and cases with complete loss, did not receive significant visual benefit from surgical decompression.

In India, presentations with advanced disease are frequent at tertiary care referral centres. We have previously noted intra-orbital and intra-cranial extension in 57 and 25 per cent of allergic fungal sinusitis presentations, respectively. This report found the prevalence of optic nerve involvement at presentation to be approximately 18 per cent. $^{6}$

The potential of allergic fungal sinusitis to cause bone erosion is well established, with a 12-fold greater likelihood of such patients having bone erosion, compared with non-allergic fungal sinusitis patients. ${ }^{15}$ Such bone erosion has been thought to be primarily caused by persistent pressure, and also by hyperaemia and inflammation of the surrounding mucosa. Incipient infiltration of the mucosa by fungal elements and consequent granulomatous reaction has also been noted. ${ }^{6}$ Optic nerve involvement in allergic fungal sinusitis may potentially be caused by either compression of the optic nerve, ${ }^{2}$ fungal toxins and consequent inflammation, ${ }^{2}$ or direct fungal infiltration. However, in the presented cases the surgical findings indicated the nerve to be clearly visible and normal, with fungal and inflammatory debris reaching up to the dura and the nerve but not causing any gross invasion. The observed immediate recovery following isolated surgical decompression indicates the probable predominant cause of nerve involvement to be extraneural compression alone.

In most of the current cases, the disease had caused significant erosion of the lateral sphenoid wall so as to cause complete baring of the optic nerve, the cavernous sinus and the internal carotid artery. Therefore, surgical decompression was accomplished by clearing of the sphenoid sinus alone. In cases in which canal erosion was restricted to the thin bone of the posterior segment, and in which the thicker bone of the anterior optic canal and the optic ring were preserved, this preserved bone was removed to achieve optic nerve decompression. The anterior part of the optic canal is its most constricted part, and surgical decompression must therefore include this segment. In cases of optic

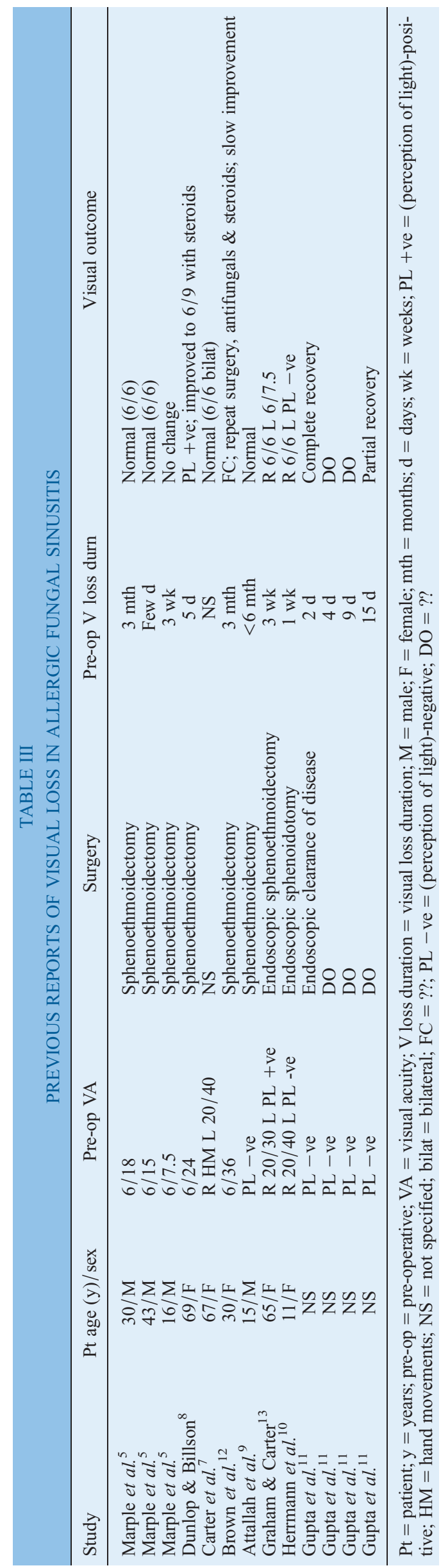


nerve injury due to other aetiologies, further incision of the optic nerve sheath is believed to enhance the results of bony decompression alone. ${ }^{14}$ However, such an incision may lead to transient cerebrospinal fluid leakage and is thus imprudent in cases of allergic fungal sinusitis, because of the potential complication of fungal meningitis.

In earlier years, the preferred approach for surgical treatment of advanced allergic fungal sinusitis was external ethmoidectomy. This had the advantage of being a two-handed approach, and also facilitated access to the laterally expanded regions of the sphenoid sinus. However, the development of endoscopic techniques in recent years has led to the ability to access most regions. The trans-pterygoid approach allows endoscopic access to the lateral sphenoid recesses.

- Visual loss is an uncommon ophthalmic manifestation of allergic fungal sinusitis

- Such visual loss is due to compression of the optic nerve rather than invasion

- Early surgical decompression has the potential to reverse such visual loss

- Prolonged visual loss and complete loss of vision are poor prognostic factors

In the current series, recovery was not noted in eyes with complete visual loss. This is in agreement with the hypothetical model of compressive visual loss. ${ }^{16}$ This model proposes that compression of the optic nerve leads to initial venous occlusion, with consequent tissue oedema and nerve fibre compression. This may worsen, either as a consequence of progressive compression, or secondary to a compartmental syndrome wherein tissue oedema initiates a progressive cycle of oedema-induced venous compression and consequent venous obstruction which induces further tissue oedema. Ongoing compression - whether due to disease or compartmental syndrome - would eventually lead to a state of arterial compression and consequent ischaemic infarction of the nerve, accompanied by complete visual loss. According to this model, venous congestion and associated partial visual loss are potentially reversible with surgical decompression, but the arterial infarction and associated complete visual loss are irreversible.

'Medical decompression' via the administration of high-dose steroids has been advocated. ${ }^{13}$ However, we are reluctant to use steroids in the setting of active fungal infestation of the sinuses. The spectrum of fungal disease in the sinuses ranges from a commensal state to fulminant invasive disease, and is influenced by both host immunity and agent pathogenicity. Presurgical administration of steroids in cases of massive fungal infestation seems imprudent. Following complete surgical removal of all fungal debris, however, we do believe that both systemic and local steroids are useful in the immediate post-surgical period to facilitate the return of the inflamed mucosa to a normal state, and also to minimise later recurrence. ${ }^{4}$

\section{Conclusion}

Visual loss is an uncommon, albeit grave, ophthalmic manifestation of allergic fungal sinusitis, and is caused by compression of the optic nerve. Early surgical decompression leads to reversal of visual loss, provided this visual loss is of recent onset and has not progressed to complete visual loss.

\section{References}

1 Safirstein BH. Allergic bronchopulmonary aspergillosis with obstruction of the upper respiratory tract. Chest 1976;70:788-90

2 Millar JW, Johnston A, Lamb D. Allergic aspergillosis of the maxillary sinus. Thorax 1981;36:710

3 Bent JP, Kuhn FA. Diagnosis of allergic fungal sinusitis. Otolaryngol Head Neck Surg 1994;111:580-8

4 Ence BK, Gourley DS, Jorgensen NL. Allergic fungal sinusitis. Am J Rhinol 1990;4:169-78

5 Marple BF, Gibbs SR, Newcomer MT, Mabry RL. Allergic fungal sinusitis induced visual loss. Am J Rhinol 1999;13:191-5

6 Thakar A, Dhiwakar M, Sarkar C, Bahadur S, Dahiya S. Allergic fungal sinusitis: expanding the clinico-pathological spectrum. Otolaryngol Head Neck Surg 2004;130:209-16

7 Carter KD, Graham SM, Carpenter KM. Ophthalmic manifestations of allergic fungal sinusitis. Am J Ophthalmol 1999; 127:189-95

8 Dunlop IS, Billson FA. Visual failure in allergic Aspergillus sinusitis: case report. Br J Ophthalmol 1988;72:127-30

9 Attallah M, Hashash M, Al-Muhaimeed H, Dousary S, al Rabah A, Kharashi S. Reversible neuropraxic visual loss induced by allergic Aspergillus flavus sinomycosis. Am J Rhinol 1999;13:295-8

10 Herrmann BW, White FV, Forsen JW Jr. Visual loss in a child due to allergic fungal sinusitis of the sphenoid. Otolaryngol Head Neck Surg 2006;135:328-9

11 Gupta AK, Bansal S, Gupta A, Mathur N. Visual loss in the setting of allergic fungal sinusitis: pathophysiology and outcome. J Laryngol Otol 2007;26:1-5

12 Brown P, Demaerel P, McNaught A, Revesz T, Graham E, Kendall BE et al. Neuro-ophthalmological presentation of non-invasive aspergillus sinus disease in the non-immunocompromised host. J Neurol Neurosurg Psychiatry 1994;57:234-7

13 Graham SM, Carter KD. Response of visual loss in allergic fungal sinusitis to oral corticosteroids. Ann Otol Rhinol Laryngol 2005;114:247-9

14 Thakar A, Tandon DA, Mahapatra AK. Surgery for optic nerve injury - should nerve sheath incision supplement osseous decompression? Skull Base 2009;19:263-71

15 Ghegan MD, Lee FS, Schlosser RJ. Incidence of skull base and orbital erosion in allergic fungal rhinosinusitis (AFRS) and non AFRS. Otolaryngol Head Neck Surg 2006;134:592-5

16 Chen YR, Breidahl A, Chang CH. Optic nerve decompression in fibrous dysplasia: indications, efficacy, and safety. Plast Reconstr Surg 1997;99:22-31

Address for correspondence:

Dr Priti Lal,

Department of Otolaryngology,

Dr Ram Manohar Lohia Hospital,

New Delhi, 110001, India

Fax: 00911123747592

E-mail: priti_lal@hotmail.com

Dr P Lal takes responsibility for the integrity of the

content of the paper

Competing interests: None declared 